\title{
La importancia de utilizar la Evaluación Formativa y Compartida en la Formación Inicial del Profesorado de Educación Física: los Proyectos de Aprendizaje Tutorado como ejemplo de buena práctica
}

\author{
The importance of using Formative and Shared Assessment in Physical Education Teacher \\ Education: Tutored Learning Projects as an example of good practice
}

Víctor M. López Pastor, Miriam Molina Soria, Cristina Pascual Arias, Juan Carlos Manrique Arribas

Universidad de Valladolid (España)

\begin{abstract}
Resumen. La finalidad de este artículo es analizar la importancia de experimentar procesos de evaluación formativa y compartida (EFyC) durante la formación inicial del profesorado de educación física (FIP-EF), si queremos que luego sean capaces de aplicar modelos más educativos de evaluación cuando ejerzan como maestros. En la formación de maestros, la EFyC tiene un doble sentido: es un sistema de evaluación pero, sobre todo, es un conocimiento y una competencia profesional a aprender. En la primera parte del artículo intentamos dar respuesta a las preguntas: ¿Qué se debe enseñar sobre evaluación en la FIP-EF? y, ¿Por qué y para qué? En la segunda parte del artículo realizamos una ejemplificación práctica de lo expuesto anteriormente. En nuestro caso explicamos cómo desarrollamos procesos de EFyC en un ejemplo de buena práctica que utilizamos habitualmente en la FIP: Los Proyectos de Aprendizaje Tutorados (PAT). Para ello detallamos las fases de que consta un PAT y explicamos cómo es fundamental ligar la metodología con procesos de EFyC para que la calidad del aprendizaje y el desarrollo de competencias profesionales sean más altos y de mayor calidad. La combinación de esta metodología con sistemas de EFyC lleva ya dos décadas acumulando evidencias de que genera buenos resultados en aprendizaje, satisfacción y autopercepción de competencias docentes.
\end{abstract}

Palabras clave. Formación Inicial del Profesorado, Educación Física, Evaluación Formativa, Evaluación Compartida.

\begin{abstract}
The purpose of this paper is to analyze the importance of using processes of formative and shared assessment (F\&SA) during Physical Education Teacher Education Programs (PETE) to prepare students to apply more educational assessment models in their future as teachers. In PETE Programs, F\&SA have a double meaning: it is an assessment system but, above all, it represents knowledge and professional skills to be learned. In the first part of the paper we try to answer the questions: (1) what should be taught about assessment in PETE Programs? (2) Why and what for? In the second part of the paper we provide a practical example. In our case, we explain how we develop F\&SA processes in an example of «good practice» that we usually employ in the PETE Program: Tutored Learning Projects (TLP). Then, we detail the phases of a TLP and explain how essential it is to link methodology with F\&SA processes and ensure that the quality of learning and the development of professional skills are higher and of higher quality. The combination of this methodology with F\&SA systems has been accumulating evidence for two decades, generating good results in learning, satisfaction, and self-perception of teaching skills.
\end{abstract}

Key-words. Pre-service Teacher Education, Physical Education, Formative Assessment, Shared Assessment.

Introducción sobre el desarrollo de sistemas de evaluación formativa y compartida (EFyC) en la formación inicial del profesorado de educación física (FIP-EF)

Hemos organizado este artículo en dos partes. En la primera realizamos una primera aproximación a la temática de la evaluación en la FIP-EF, intentando responder a preguntas como las siguientes: ¿Por qué hay que superar los modelos tradicionales de evaluación-calificación en FIP-EF? ¿Qué importancia tiene experimentar procesos de EFyC durante la FIP-EF y cuáles son sus ventajas? ¿Qué debe de enseñarse sobre evaluación en la FIP-EF? ¿Para qué hay que experimentar y enseñar sistemas de EFyC en FIP (la necesaria trasferencia a la práctica docente)?

En una segunda parte presentamos un ejemplo concreto de una «buena práctica» en FIP-EF y el papel que la evaluación formativa y compartida juega en todo el proceso para garantizar un mejor aprendizaje del alumnado y un mejor funcionamiento del proceso de enseñanza-aprendizaje.

¿Por qué hay que superar los modelos tradicionales de evaluación-calificación? El reto de pasar de la «cultura del examen» a la «cultura de la evaluación»

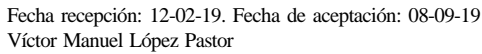

En la docencia universitaria ha predominado durante muchos años la figura del profesor y las horas de docencia como eje central, normalmente en base a la metodología didáctica de «lección magistral». Este paradigma formativo «Centrado en el profesor» se ha reproducido durante tantos años que ha llegado a convertirse en el paradigma tradicional. El cambio hacía un paradigma educativo centrado en el estudiante parece estar muy relacionado con la introducción y generalización del paradigma centrado en el «desarrollo de competencias» (Benito \& Cruz, 2003; Zabalza, 2002), que afecta poderosamente a dos elementos curriculares: la metodología didáctica y la evaluación (López-Pastor, 2009).

Por tanto, uno de los retos fundamentales a los que se enfrenta la FIP-EF es la superación del denominado «Aprendizaje Bancario» y el avance hacia el denominado modelo de «Aprendizaje Dialógico», que es el paradigma de aprendizaje que mejor permite el logro de los aprendizajes complejos y las competencias que requiere una formación universitaria. Freire (1990) explica a fondo la diferencia entre los dos conceptos («Aprendizaje dialógico vs. Aprendizaje bancario») y sus implicaciones prácticas. Flecha y Puigvert (1998) también profundizan en la temática del «aprendizaje dialógico», sus bases científicas y/o ejemplificaciones prácticas.

A lo largo de este trabajo veremos cómo el modelo de EFyC rompe considerablemente con los extendidos modelos de aprendizaje bancario, en los que se considera que la tarea docente consiste en enseñar los hechos, conceptos y pro- 
cedimientos de la asignatura, como si su finalidad fuera verter el conocimiento en la cabeza de sus alumnos hasta completar la medida adecuada, proporcionando las respuestas correctas que deben ser recordadas y demostradas en el examen final. El rol de la evaluación en este modelo de aprendizaje se reduce a la medición final de resultados, normalmente mediante exámenes finales, que se traducen en una única calificación final. En estos modelos la evaluación suele ser sinónimo de examen y calificación. Se trata de una tradición que parece estar cambiando, en la que el papel del alumnado ha cobrado mayor importancia, situándose en el centro del proceso de aprendizaje. En este sentido, Dochy, Segers y Dierick (2002) defienden que la universidad europea tiene que comenzar a superar la «cultura del examen» y avanzar más en la «cultura de la evaluación». La literatura especializada sobre esta idea de avanzar en la «cultura de la evaluación» muestra numerosas experiencias de éxito de EFyC en los últimos años en Educación Superior (Knight, 2005; López-Pastor, 2009; López-Pastor y\& Pérez-Pueyo, 2017; Santos, Martínez, \& López, 2009).

La importancia de experimentar procesos de evaluación formativa y compartida (EFyC) durante la formación inicial del profesorado de educación física (FIP-EF)

La EFyC constituye una alternativa eficaz para avanzar hacía modelos de aprendizaje dialógico y la cultura de la evaluación en la FIP-EF. La evaluación formativa busca generar procesos de mejora y aprendizaje en tres sentidos: (a) optimizar los procesos de aprendizaje y producciones del alumnado; (b) perfeccionar la práctica docente; y, (c) reconducir los procesos de enseñanza-aprendizaje que se llevan a cabo en el aula, tanto durante el propio desarrollo de la asignatura como tras la finalización del mismo, de cara a programar el siguiente curso. Este proceso de mejora y optimización se realiza desde una perspectiva humanizadora, sin una finalidad calificadora, (López-Pastor, 2009; López-Pastor \& PérezPueyo, 2017) y la utilización correcta del feedback es un punto clave.

Nuestra propuesta une y relaciona el concepto de «evaluación formativa» con el de «evaluación compartida», dado que entendemos que la evaluación formativa debe facilitar y dar también importancia a la participación del alumnado en los procesos de evaluación, a través de diferentes técnicas: la autoevaluación, la evaluación entre iguales y la evaluación dialógica (López-Pastor, 2009). El concepto de «evaluación compartida» hace referencia a los procesos de diálogo que mantiene el profesor con su alumnado sobre la evaluación de los aprendizajes y los procesos de enseñanza-aprendizaje que tienen lugar. Este tipo de diálogos pueden ser individuales o grupales y suelen estar basados en procesos previos de autoevaluación o evaluación entre iguales, (LópezPastor, 2012, p. 121). Desarrollar procesos de evaluación compartida en el aula está muy relacionado con la cesión de responsabilidad en el aula y con la implicación del alumnado en la toma de decisiones que afectan a los procesos de aprendizaje. Las experiencias innovadoras han demostrado que implicar al alumnado en los procesos evaluativos genera una mejora en sus propios procesos de aprendizaje (Brown, 2015).

Hay varias razones que justifican la utilización de sistemas de EFyC en FIP-EF, apoyadas por evidencias recogidas en los estudios llevados a cabo en los últimos diez años (Cañadas, Santos-Pastor \& Castejón, 2018; Fraile, Aparicio, Asún, \& Romero-Martín, 2018; Gallardo \& Carter, 2016; Hamodi, Moreno-Murcia, \& Barba, 2018; López-Pastor, 2008, 2009; López-Pastor, Pérez-Pueyo, Barba \& Lorente-Catalán, 2016; Romero, Asún \& Chivite, 2016; Romero-Martín, Castejón-Oliva, López-Pastor \& Fraile-Aranda, 2017). Las principales son las siguientes: (1) porque sirve para aprender más y mejor; (2) porque desarrolla mejor las competencias docentes en FIP; (3) porque ayuda a que el alumnado se centre más en su proceso de aprendizaje y asuma su responsabilidad en el mismo; (4) porque genera más éxito educativo y mejor rendimiento académico; (5) porque es una competencia básica de todo maestro, que normalmente se adquiere más por experimentación práctica que por estudio teórico; (6) porque facilita que haya una mejor transferencia entre lo aprendido en la FIP y la práctica educativa en las escuelas.

A lo largo de los primeros apartados del artículo vamos a ir desarrollándolas, aportando los estudios y evidencias acumulados hasta el momento sobre estas temáticas.

Los sistemas de EFyC han sido puestos en práctica, investigados, evaluados y perfeccionados en FIP-EF durante las últimas décadas, demostrando su viabilidad y adecuación. Existen estudios que demuestran que la EFyC es idónea y factible en la FIP (Cañadas, Santos-Pastor \& Castejón, 2018; Gallardo \& Carter, 2016; López-Pastor, Pérez-Pueyo, Barba \& Lorente-Catalán, 2016; Romero, Asún \& Chivite, 2016), ya que presenta una estrecha relación con la adquisición de competencias profesionales específicas, mediante la implicación del alumnado en los procesos evaluativos.

Otros estudios se centran en demostrar la eficacia que tiene la utilización de sistemas de EFyC en la FIP, en aspectos como: (a) el aprendizaje (Biggs, 2005; Knight, 2005; López-Pastor, 2009); (b) la satisfacción (Atienza, ValenciaPeris, Martos-García, López-Pastor \& Devís-Devís, 2016; Romero-Martín, Castejón-Oliva, López-Pastor \& FraileAranda, 2017); (c) el rendimiento académico y/o desarrollo de competencias docentes (Fraile, Aparicio, Asún, \& Romero-Martín, 2018; Hamodi, Moreno-Murcia, \& Barba, 2018).

En un estudio con una muestra muy amplia de alumnado universitario de FIP-EF de toda España, Romero-Martín, Castejón-Oliva, \& López-Pastor (2015) encuentran un alto grado de satisfacción con la utilización de sistemas de evaluación formativa. En un estudio más local, con alumnado de último curso de grado en FIP, Silva y López-Pastor (2015) muestran que, en general, el alumnado muestra una valoración general positiva, especialmente respecto a los efectos en su aprendizaje y en su rendimiento académico. También consideran que ayuda a organizar mejor el trabajo y a realizar un reparto equilibrado del mismo a lo largo del cuatrimestre, evitando saturaciones finales. Similares resultados aparecen también en Gallardo, López-Pastor y Carter (2018) en FIP, donde el alumnado valora muy positivamente la utilización de sistemas de EFyC en sus estudios, considerando que tiene muchas e importantes ventajas y pocos inconvenientes. Muchos de estos estudios muestran que tanto el alumnado como el profesorado valoran positivamente su uso en la FIP, aunque parecen existir pequeñas diferencias en función del curso, de modo que, aunque todo el alumnado valora muy positivamente la ventaja de utilizar sistemas de 
EFyC en la FIP, las valoraciones son más altas en los cursos superiores que en los inferiores. Por tanto, parece que la experiencia previa (cursos más altos) y/o una mayor madurez influyen positivamente en la mejor percepción de las ventajas de los sistemas de EFyC en la FIP; mientras que el alumnado de cursos iniciales presenta mayor dificultad para comprender y aprovechar los sistemas de EFyC (Gallardo \& Carter, 2016; Gallardo et al., 2018; Hortigüela-Alcalá, PérezPueyo, \& Abella, 2015; Manrique-Arribas, Vallés \& Gea, 2012).

En los últimos quince años se están generando numerosos estudios sobre la percepción que tiene el alumnado y los egresados sobre cómo los sistemas de EFyC vividos en su FIP les han ayudado a adquirirla. En un estudio realizado hace ya casi una década, Manrique, López, Monjas, \& Real (2010) encuentran que un porcentaje relativamente alto del alumnado de FIP considera que la utilización de procesos de EFyC en algunas asignaturas a lo largo de su FIP les ha ayudado en el desarrollo de sus competencias profesionales. Similares valoraciones aparecen también en investigaciones más actuales (Gallardo, Carter \& López-Pastor, 2019; Romero-Martín, Fraile-Aranda, López-Pastor, \& CastejónOliva, 2014; Romero et al., 2017; Silva \& López-Pastor, 2015).

En varios estudios el alumnado indica que estos sistemas de evaluación contribuyen a mejorar sus competencias éticas, de trabajo en equipo y sus habilidades interpersonales, así como sus competencias docentes, manifestando que esto se debe principalmente a la interacción profesor-estudiante (Hortigüela-Alcalá et al., 2015; Romero-Martín et al., 2017).

Con respecto a la auto-percepción de competencias profesionales adquiridas durante las asignaturas que utilizan un sistema de EFyC en la FIP, los resultados indican unos valores elevados en tres escalas de competencias diferentes («Transversales», «Docentes Generales» $\mathrm{y}$ «Docentes Específicas de Educación Física»). También indican claramente que la única escala que muestra puntuaciones más altas en el post-test es la de auto-percepción de competencia docente específica en EF, resultados lógicos dado que se trata de asignaturas específicas de EF. Por ejemplo, Gallardo et al. (2018) y Molina y López-Pastor (2019) encuentran resultados satisfactorios en asignaturas del Grado en Educación Infantil, así como Gutiérrez-García (2018) en el Grado en Ciencias de la Actividad Física y del Deporte.

Por otra parte, la mayoría de los estudios realizados en los últimos años muestra que habitualmente estos sistemas de evaluación influyen positivamente en el rendimiento académico y en la disminución de los niveles de fracaso y abandono de estudios (Fraile, López, Castejón, \& Romero, 2013; Romero et al., 2014, 2017). Hay explicaciones lógicas para estos resultados. La evaluación formativa es un elemento fundamental para que el profesorado y alumnado hagan más efectivo el proceso de aprendizaje, dado que permite retroalimentar el aprendizaje y orientarle para que se consigan los objetivos planteados. Además, hacer partícipe al alumnado del proceso de evaluación incide no sólo en su aprendizaje, sino también en su autonomía personal, en su autorregulación y pensamiento crítico y autocrítico, así como en la mejora del clima del aula (Hamodi, López-Pastor y LópezPastor, 2014; López-Pastor, 2008, 2009).

\section{¿Por qué debe utilizarse la EFyC en la FIP-EF? El doble sentido de la evaluación y la metodología en la FIP: procesos didácticos vividos y conocimiento y competencia profesional a aprender}

Consideramos que en la FIP-EF es doblemente importante utilizar y desarrollar sistemas de evaluación formativa en las diferentes asignaturas, así como favorecer la participación del alumnado en los sistemas y procesos de evaluación. La primera razón es general: este tipo de sistemas suele generar mejores procesos de aprendizaje y el desarrollo de una mayor capacidad de aprendizaje permanente (aprender a aprender). La segunda es específica de la FIP y especialmente relevante: es difícil que un maestro pueda poner en práctica en el futuro sistemas y procesos de evaluación formativa y compartida si no los ha vivido y experimentado durante su formación inicial. Esto es aún más importante cuando toda nuestra formación actual está enfocada al desarrollo de competencias profesionales.

La pregunta que deberíamos plantearnos todas las personas que nos dedicamos a la FIP es la siguiente: ¿Cómo es posible que el profesorado en formación inicial desarrolle buenas competencias en evaluación si nunca utiliza y experimenta procesos, técnicas e instrumentos de evaluación formativa durante dicha formación inicial; o si nunca ha tenido que enfrentarse a instrumentos y procesos de autoevaluación, evaluación entre iguales o evaluación compartida; o nunca ha tenido que elaborar instrumentos de evaluación específicos para situaciones concretas de aprendizaje?

En este sentido, Fullan (1991) defiende que en la FIP es fundamental predicar con el ejemplo. Existen algunos trabajos (Fernández-Pérez, 1989; Hernández-Álvarez, 2000; LópezPastor, 2009, 2013; Lorente-Catalán \& Kirk, 2013, Palacios \& López-Pastor, 2013; Tejedor, 1998; Tonucci, 2010) que remarcan la importancia que tiene en la FIP la coherencia interna entre la metodología de enseñanza-aprendizaje utilizada y los sistemas de evaluación que la complementan, denuncian las graves consecuencias que tiene la habitual incoherencia que se repite año tras año en el ámbito de la FIP, y que consiste en recibir discursos sobre cómo hacer la evaluación en la práctica educativa que son completamente contrarios con la evaluación que se lleva a cabo en dichas asignaturas (ver figura 1 ).

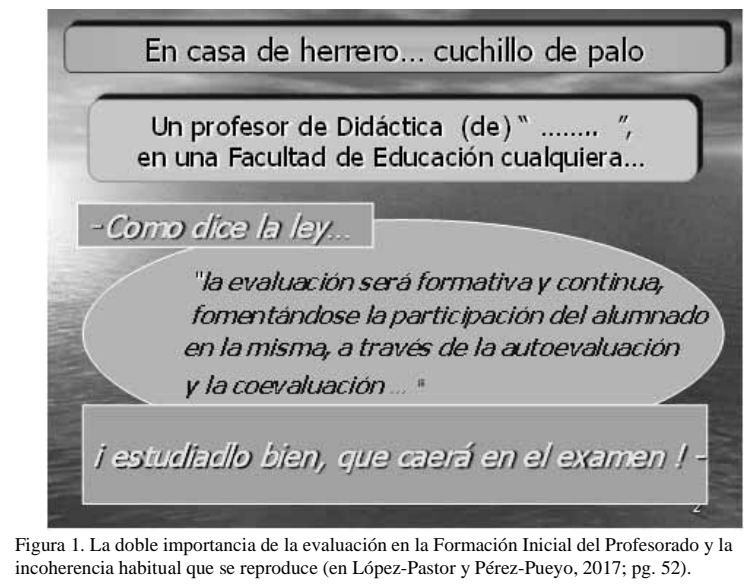

Una pregunta importante puede ser: ¿Están evolucionando los sistemas de EFyC que se utilizan en la FIP? Y la 
respuesta, afortunadamente, es que sí que están evolucionando y a más velocidad de lo que habían evolucionado en los anteriores 50 años. Parece que un punto clave ha sido la implantación progresiva del Espacio Europeo de Educación Superior (EEES) en la universidad española. Por ejemplo, un estudio llevado a cabo por López-Pastor, Manrique y Vallés (2011) comprueba que la implantación de los nuevos estudios de Grado parece haber generado cambios significativos en los procesos de evaluación del alumnado en la FIP. Los tres principales son: (a) ofrecer al alumnado diferentes vías de aprendizaje y evaluación, dando prioridad a la vía de evaluación continua; (b) diversificar el sistema de calificación del aprendizaje del alumnado, perdiendo mucho peso el examen (final o parcial), para repartirlo con el resto de evidencias y actividades de aprendizaje realizadas; (c) promover la participación del alumnado en el proceso de evaluación (autoevaluación, evaluación entre iguales y/o evaluación compartida). Estos cambios parecen ser una continuación de líneas de innovación ya desarrolladas previamente a través de experiencias-piloto.

Este tipo de resultados concuerda con los encontrados por Palacios y López (2013) sobre las tres grandes tipologías de profesorado universitario en la FIP, en función de si están desarrollando sistemas de evaluación formativos y compartidos («profesorado innovador»), si siguen reproduciendo modelos tradicionales («profesorado tradicional») o si están introduciendo algunos cambios en los sistemas de evaluación, pero no de forma clara y completa («profesorado ecléctico»). El factor más importante para formar parte del grupo de «profesorado innovador» parece ser la participación en actividades de formación permanente sobre docencia universitaria, especialmente seminarios y proyectos de innovación docente centrados en la mejora de la propia práctica docente. Por otra parte, parece que es todavía una minoría el profesorado que utiliza de forma habitual sistemas de evaluación formativa y que diferencia claramente entre el sistema y proceso de evaluación y el sistema de calificación final.

¿Para qué hay que utilizar EFyC en FIP? La trasferencia entre lo vivido en FIP y el ejercicio profesional como docentes

Algunas personas expertas en el tema resaltan la importancia que tiene utilizar en la FIP metodologías activas y sistemas de EFyC si queremos que la escuela vaya cambiando y superando poco a poco las metodologías tradicionales de aprendizaje y de evaluación (López-Pastor, 2009, 2013; Lorente-Catalán \& Kirk, 2013, Palacios \& López-Pastor, 2013).

Recientes estudios están comprobando que existe cierta trasferencia entre la utilización de sistemas de EFyC durante la FIP y el desarrollo y aplicación de este tipo de sistemas en su vida profesional cuando los estudiantes acceden posteriormente a puestos de trabajo como maestros (Barrientos, López-Pastor, \& Pérez-Brunicardi, 2019; Hamodi, López-Pastor, \& López-Pastor, 2017; Lorente-Catalán, López-Pastor \& Kirk, 2018; Molina \& López-Pastor, 2019). Se trata de un proceso lógico, dado que diferentes investigaciones indican que el profesorado tiende a utilizar como métodos evaluativos los que fueron utilizados con ellos cuando eran alumnos. Dicho de otra forma, los estudiantes de FIP tienden a reproducir en su posterior trabajo como maestros de EF los mismos métodos que han vivido como alumnos durante su paso por secundaria y FIP (Fullan, 1991; Hamodi et al., 2017; Lorente-Catalán \& Kirk, 2013; Tonucci, 2010).

Parece necesario realizar una mayor investigación sobre el uso de estos tipos de evaluación en FIP, así como de la importancia de vivir este tipo de sistemas de evaluación durante su formación inicial si queremos que el profesorado de EF sea luego capaz de ponerlo en práctica en las escuelas. En esta línea, Molina y López-Pastor (2017, 2019) comprueban que muchos maestros de EF que utilizan sistemas de EFyC actualmente han vivido este tipo de sistemas durante su FIP.

Entendemos que estos procesos de trasferencia entre los sistemas de evaluación vividos durante la FIP y la práctica educativa puede tener una gran importancia a la hora de lograr una mayor difusión e implantación de este tipo de «buenas prácticas» en la escuela (Córdoba, López-Pastor \& Sebastiani, 2018; Hamodi et al., 2017; Lorente-Catalán et al., 2018). Sobre los problemas de trasferencia, Lorente-Catalán \& Kirk $(2013,2016)$ señalan que aunque en el contexto inglés se está aplicando el modelo de «evaluación para el aprendizaje» en gran parte de la FIP-EF, dichas prácticas no están extendidas en la EF escolar. Parece que el profesorado en ejercicio considera que esas prácticas que realizaron en su FIP no son trasferibles a las escuelas. En este estudio, los estudiantes mostraron su intención de utilizar la evaluación para el aprendizaje en su futura práctica profesional, aunque investigaciones recientes parecen mostrar que los profesores noveles encuentran barreras en la práctica educativa para realizarlas, dado que no se utilizan de forma habitual en la EF escolar, por ejemplo en Lorente-Catalán et al., 2018. Sin embargo, el profesorado novel español que ha vivido sistemas de EFyC durante su FIP suele aplicar estos sistemas en su práctica actual como maestros, aunque es cierto que a veces encuentran obstáculos para ponerlos en práctica (Hamodi et al., 2017; Molina \& López-Pastor, 2017).

Por otra parte, no siempre es suficiente con haber vivido unas adecuadas experiencias en FIP. En muchos casos es necesaria también una adecuada formación permanente para seguir evolucionando profesionalmente y aprender a utilizar sistemas de EFyC en el aula. Incluso, en muchas ocasiones, la formación permanente adecuada tiene la suficiente fuerza como para que el profesorado que no tuvo experiencias de metodologías activas ni EFyC durante su FIP-EF haya trasformado radicalmente su creencias y su forma de trabajar, para ir avanzando hacía este tipo de metodologías en su práctica educativa (Barrientos et al., 2019; Córdoba et al., 2018; Molina y López-Pastor, 2017). Por tanto, la evidencia muestra que la mejora profesional de los docentes depende en gran medida de su capacidad para desarrollar acciones colaborativas con otros profesores y con el propio alumnado, con los que intercambia conocimientos y experiencias.

\section{Los Proyectos de Aprendizaje Tutorado (PAT) como ejemplo de buena práctica de evaluación formativa y com- partidaenFIP-EF}

En esta segunda parte presentamos un ejemplo práctico de lo expuesto anteriormente. Se trata de una «buena práctica» que utilizamos habitualmente en la FIP: los «Proyectos 
de Aprendizaje Tutorados» (PAT). Para ello detallamos qué es el PAT, cómo se lleva a cabo y la importancia de ligar esta metodología con procesos de EFyC para que la calidad del aprendizaje y el desarrollo de competencias profesionales sean más altos.

\section{Qué es el PAT}

El PAT consiste en una actividad de aprendizaje grupal que, normalmente, implica la realidad de una práctica (real o simulada), donde se ponen en juego diferentes competencias profesionales. La elaboración del proyecto implica la realización de una serie de borradores que se revisan con el tutor, recibiendo feedback y mejorándolos, hasta que tienen la calidad suficiente para ponerlos en práctica con el resto de sus compañeros. De esta forma se asegura una calidad mínimamente aceptable en la puesta en práctica y en la documentación a entregar a sus compañeros. Se trata de una metodología didáctica muy potente en la FIP, que ha demostrado ser una forma muy eficaz de trabajo teórico-práctico y de desarrollo de competencias profesionales (Barba-Martín, López-Pastor, Manrique-Arribas, Gea-Fernández, \& Monjas-Aguado, 2010; Gutiérrez-García, 2018; Manrique et al., 2010). Algunos estudios hacen referencia a la utilización de metodologías activas y su combinación con procesos de EFyC, especialmente en lo relativo a la utilización sistemática de los PAT, por los buenos resultados obtenidos en todos los cursos y en todas las asignaturas de FIP (Barba \& LópezPastor, 2017; Estevan, Molina, García, \& Martos, 2018; LópezPastor \& Pérez-Pueyo, 2017; López-Pastor, Hamodi \& LópezPastor, 2016). Este tipo de metodologías se pueden combinar con otros instrumentos, como las rúbricas y/o el uso de carpetas colaborativas, como forma de cerrar el proceso de aprendizaje y la obligada calificación final (López-Pastor, 2011; López-Pastor \& Pérez-Pueyo, 2017).

\section{Cómo combinamos los procesos de evaluación formativa y compartida con los PAT en la FIP-EF}

En la Facultad de Educación de Segovia llevamos unos 20 años utilizando la metodología de los PAT, en numerosas asignaturas de los diferentes Grados que impartimos (Educación Primaria, Educación Infantil, Doble grado), porque han demostrado ser una forma muy eficaz de trabajo teóricopráctico y para la adquisición de competencias profesionales. Utilizamos el PAT como una actividad grupal. Cada grupo de alumnos prepara una sesión práctica y un marco teórico de un tema a elegir dentro de una lista de propuestas prácticas de intervención en EF. El proceso de EFyC se da durante todo el proceso de elaboración y puesta en práctica del PAT. Éste comienza en las tutorías previas entre el profesorado y el alumnado para la revisión y mejora de los documentos y el plan de actuación, posteriormente se valora la puesta en práctica y se termina en los diferentes informes finales que elabora cada grupo, hasta cerrar el proceso de forma satisfactoria. La EFyC demuestra ser un proceso clave para lograr mejores aprendizajes y conseguir una buena calidad de los trabajos.

El proceso de realización de un PAT suele seguir las fases mostradas en la Tabla 1.

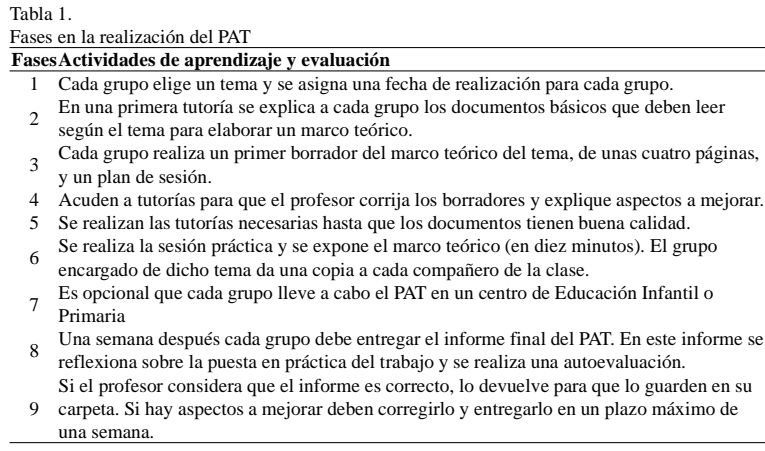

El sistema general de evaluación formativa y continua que solemos utilizar en varias asignaturas de FIP puede apreciarse en la Tabla 2. El punto de partida son los principales tipos de actividades de aprendizaje que se pueden desarrollan a lo largo de todo el cuatrimestre; a partir de ahí, se explican los instrumentos de evaluación que se utilizan, cómo se hace el proceso de evaluación formativa y qué peso tiene cada actividad de aprendizaje en la evaluación. Pueden encontrarse ejemplos más detallados en López-Pastor (2008, 2011).

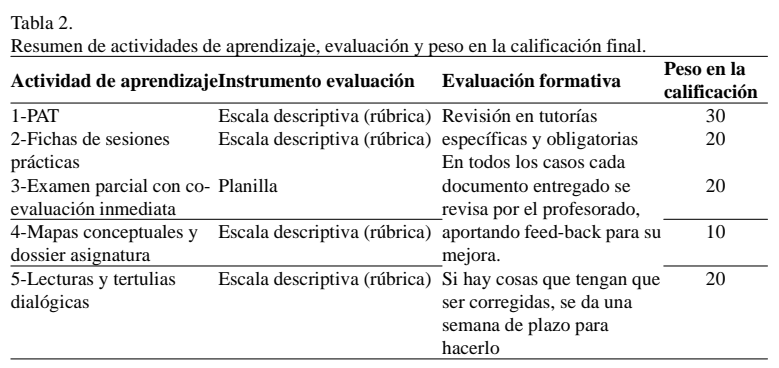

Un aspecto importante a tener siempre en cuenta es que la evaluación y la calificación son los elementos curriculares que más condicionan los procesos de aprendizaje del alumnado. En este sentido, Biggs (2005) explica que la evaluación condiciona qué y cómo aprende el alumnado, lo que probablemente sea uno de los condicionantes más poderosos de los procesos de aprendizaje que se desarrollan. Por su parte, Gibbs (2003) afirma que la evaluación es el arma más poderosa que tienen los profesores para influir en el modo en el que los estudiantes responden a las asignaturas y orientan el aprendizaje. Por tanto, si utilizamos sistemas tradicionales de evaluación-calificación, lo normal es que el alumnado se limite a estudiar solo al final, los días anteriores al examen. En cambio, si utilizamos sistemas de EFyC, como el que presentamos en el ejemplo de la tabla 2, el alumnado repartirá el trabajo a lo largo de todo el cuatrimestre y centrará su esfuerzo en las diferentes actividades de aprendizaje que forman parte del sistema de evaluación. Dado que consideramos el PAT como una de las actividades de aprendizaje más importantes en la asignatura, su peso en la calificación es el mayor de todos.

En las Tablas 3, 4 y 5 presentamos los instrumentos de evaluación que solemos utilizar para los procesos de autoevaluación y evaluación compartida que llevamos a cabo a lo largo de la elaboración del PAT.

La Tabla 3 es una escala graduada que, en nuestro caso, solemos utilizar como proceso de autoevaluación que realiza el grupo antes de ir a la tutoría con el borrador del PAT, publicada por Pérez-Pueyo, Barba, López-Pastor, \& Lorente- 
Catalán (2017). En la tutoría se revisa el borrador del PAT, delante del grupo de alumnos, anotando y explicando los cambios y correcciones que deben realizar. El alumnado dispone desde el principio de una escala graduada para poder realizar una autoevaluación del trabajo. Ese instrumento también puede ser utilizado al finalizar el PAT, para dar el salto entre el mes largo de trabajo y aprendizaje que supone toda la elaboración del PAT y la calificación final correspondiente en esta actividad de aprendizaje.

\begin{tabular}{|c|c|c|}
\hline $\begin{array}{l}\text { Valoración } \\
\text { subjetiva }\end{array}$ & $\begin{array}{l}\text { Horquilla } \\
\text { numérica }\end{array}$ & Criterios \\
\hline $\begin{array}{l}\text { MAL/ Necesita } \\
\text { mejorar mucho }\end{array}$ & Hasta 4,9 & $\begin{array}{l}\text { El contenido no cumple con el objetivo del trabajo, no presenta } \\
\text { de forma clara y explícita las intenciones del trabajo, no tiene } \\
\text { índice, incurre en numerosos plagios, numerosas faltas de } \\
\text { ortografía, problemas graves de citación y en referencias. }\end{array}$ \\
\hline $\begin{array}{l}\text { REGULAR/ } \\
\text { Aunque supera lo } \\
\text { mínimo exigible, } \\
\text { necesita mejoras } \\
\text { importantes }\end{array}$ & $5-5,9$ & $\begin{array}{l}\text { El contenido solo aborda tangencialmente los objetivos, poca } \\
\text { concreción y claridad en el desarrollo de los objetivos del } \\
\text { trabajo, incoherencias entre las intenciones y el desarrollo, } \\
\text { conclusiones con incoherencias, problemas de expresión y } \\
\text { redacción con algunas faltas de ortografía, pocas citas y } \\
\text { referencias con algunos problemas de formato, aspectos formales } \\
\text { y de presentación poco cuidados }\end{array}$ \\
\hline $\begin{array}{l}\text { BIEN/ Comienza a } \\
\text { tener los rasgos de } \\
\text { un trabajo pero } \\
\text { debe cuidar los } \\
\text { aspectos que lo } \\
\text { harán tener un valor } \\
\text { destacado }\end{array}$ & $\begin{array}{l}6-6.9 \\
\end{array}$ & $\begin{array}{l}\text { El contenido se ajusta al objetivo, aunque le falta } \\
\text { fundamentación, falta claridad en el trabajo, dificultad para } \\
\text { diferenciar entre el discurso del autor y las referencias, tras las } \\
\text { revisiones del profesor no se han solucionado las dificultades, el } \\
\text { lenguaje es apropiado per con incorrecciones, no están presentes } \\
\text { todas las citas en las referencias pequeños errores de formato en } \\
\text { citas y referencias, el trabajo está bien organizado pero tiene } \\
\text { alguna falta de ortografía. }\end{array}$ \\
\hline $\begin{array}{l}\text { MUY BIEN/ El } \\
\text { trabajo va por buen } \\
\text { camino pero debe } \\
\text { cuidar los detalles }\end{array}$ & 7-7.9 & $\begin{array}{l}\text { El contenido se ajusta a los objetivos pero no tiene una visión } \\
\text { crítica, la temática y las intenciones son adecuadas, las } \\
\text { conclusiones se adecuan al contenido del trabajo, en referencias } \\
\text { y citas hay pequeños fallos no generalizados, hay un número } \\
\text { considerable de citas de revistas científicas, el estilo es correcto } \\
\text { y no presenta falta de ortografía }\end{array}$ \\
\hline $\begin{array}{l}\text { MUY BUENO/ Si } \\
\text { se cuidan los } \\
\text { pequeños detalles } \\
\text { será brillante }\end{array}$ & 8- 8.9 & $\begin{array}{l}\text { Los objetivos están desarrollados en el desarrollo del trabajo y } \\
\text { en las conclusiones, hay cierta crítica pero no es clara, la } \\
\text { escritura es clara y con buen puntuación, algo menos de la mitad } \\
\text { de las citas son de revistas científicas }\end{array}$ \\
\hline $\begin{array}{l}\text { EXCELENTE/ } \\
\text { Enhorabuena }\end{array}$ & $9-10$ & $\begin{array}{l}\text { El trabajo desarrolla completamente los objetivos, es coherente y } \\
\text { crítico, expone fortalezas y debilidades, se expone claramente la } \\
\text { aportación personal, está bien cuidado en redacción y aspectos } \\
\text { formales, la mitad de las citas provienen de revistas científicas }\end{array}$ \\
\hline
\end{tabular}

Tabla 4.
Ficha para la evaluación reflexiva sobre la puesta en práctica del PAT.
\begin{tabular}{l} 
NOMBRE DEL P.A.T.: \\
\hline \multicolumn{1}{c}{ Posibles aspectos a evaluar } \\
\hline Diferencias entre lo planificado y lo ocurrido \\
\hline Reuniones internas llevadas a cabo y asistencia \\
\hline Reuniones con el profesor y breve comentario de lo realizado \\
\hline Problemas que surgen durante el proceso y como se solucionan \\
\hline Valoración del proceso de aprendizaje que se lleva a cabo a lo largo del PAT \\
\hline Otros aspectos a comentar
\end{tabular}

La Tabla 4 es una ficha de autoevaluación reflexiva sobre la sesión (o sesiones) de la puesta en práctica. Se trata de que el alumnado realice un proceso de reflexión y evaluación del funcionamiento del PAT según los criterios y las variables a considerar. Esta ficha se entrega dentro del informe final del PAT, justo a continuación del narrado sobre lo que ocurrió en la puesta en práctica.

La Tabla 5 es una ficha de autoevaluación que el alumnado tiene que entregar junto con el informe final del PAT, una semana después de su aplicación práctica. En ella el grupo de alumnos realiza una autoevaluación grupal sobre la calidad del trabajo generado, siguiendo los criterios establecidos al comienzo del proceso, así como de la aportación de cada persona en el PAT. Tras corregir el trabajo, el profesor aporta su propia valoración, escribe el feedback extra que considere necesario y, si es necesario, corregir el trabajo y entregar una nueva versión o si tienen que hablar con él. Por tanto, sirve para realizar un proceso doble: una autoevaluación grupal más una evaluación compartida con el profesor.

\section{Resultados}

La evaluación del funcionamiento del PAT se lleva a cabo a través de un cuestionario anónimo para el alumnado, previamente validado (Castejón, Santos, \& Palacios, 2015), que utiliza una escala tipo Likert (1-5).

Las valoraciones son muy altas en la mayoría de los ítems (media aritmética entre 3.40 y 4.50). La experiencia de buena práctica del PAT tiene buena acogida y satisfacción en el alumnado. El alumnado considera que el grado de dificultad de la experiencia no ha sido difícil y tiene mucha utilidad como futuros docentes, aunque sí consideran que ha tenido mucha carga de trabajo. Asimismo, el profesorado también realiza una valoración de la experiencia de buena práctica. Los docentes están de acuerdo en que esta experiencia es innovadora, efectiva, se mantiene en el tiempo, produce efectos duraderos y es aplicable a otros contextos.

Los principales resultados obtenidos durante esta experiencia en los últimos 10 años, sobre la evaluación de la asignatura, muestran una elevada satisfacción del alumnado con este tipo de procesos de aprendizaje y evaluación. El alumnado considera que los sistemas de EFyC ofrecen alternativas a todos los estudiantes, están centrados en el proceso de enseñanza-aprendizaje, permiten aprendizajes funcionales y generan aprendizajes significativos gracias a la retroalimentación, además de que la calificación es considerada más justa. Sin embargo, opinan que estos sistemas tienen mucha carga de trabajo y que exige un mayor esfuerzo y participación en la propia evaluación (autoevaluación) (Barba-Martín et al., 2010; Barba \& López-Pastor, 2017; López-

\begin{tabular}{|c|c|c|c|c|c|c|c|c|c|}
\hline \multicolumn{4}{|l|}{$\begin{array}{l}\text { Componentes Del Grupo: } \\
\text { 1-Coordinador-a: } \\
\text { 2-Secretario-a: }\end{array}$} & \multicolumn{5}{|l|}{\begin{tabular}{|l|} 
3-Vocal: \\
4-Vocal: \\
5-Vocal:
\end{tabular}} & \multirow{3}{*}{\begin{tabular}{|c|}
$\begin{array}{l}\text { Fecha de entrega } \\
\text { Tipo de trabajo: }\end{array}$ \\
Observaciones
\end{tabular}} \\
\hline & & \multicolumn{6}{|c|}{ Componentes del grupo } & \multirow[t]{2}{*}{ Calidad del documento } & \\
\hline \multicolumn{2}{|l|}{ Aspectos a evaluar } & 1 & 2 & 3 & 4 & 5 & 6 & & \\
\hline \multicolumn{10}{|l|}{ Aprendizaje } \\
\hline \multicolumn{10}{|l|}{ Organización y presentación } \\
\hline \multicolumn{10}{|l|}{ Utilización bibliografía y citas } \\
\hline \multicolumn{10}{|l|}{ Desarrollo del contenido } \\
\hline \multicolumn{10}{|l|}{ Aportaciones propias - calidad del análisis } \\
\hline \multirow{2}{*}{\multicolumn{10}{|c|}{ - Tiempo de elaboración (en minutos) }} \\
\hline \multicolumn{2}{|l|}{ Otros aspectos: } & & & & & & & & \\
\hline \multirow{2}{*}{\multicolumn{10}{|c|}{ Aportación de cada uno al trabajo grupal }} \\
\hline \multirow{2}{*}{\multicolumn{5}{|c|}{ Acta de reuniones: }} & & & & & \\
\hline NIVEL A & NIVEL B & & & & \multicolumn{4}{|c|}{ NIVEL C } & NIVEL D \\
\hline $\begin{array}{l}\text { 1-Marco teórico muy bien elaborado. } \\
\text { 2-Alto dominio de las competencias } \\
\text { docentes durante el desarrollo de la sesión } \\
\text { práctica; } \\
\text { 3-Muy buena presentación oral del marco } \\
\text { teórico (clara, sin leer, mirar a todos, etc.). } \\
\text { 4-Informe final bien presentado y } \\
\text { elaborado y buen análisis de la sesión }\end{array}$ & \multicolumn{4}{|c|}{\begin{tabular}{|l} 
1-Marco teórico bien elaborado. \\
2-Buen dominio de las competencias \\
docentes durante el desarrollo de la sesión \\
práctica; \\
3-Buena presentación oral del marco \\
teórico (clara, sin leer, mirar a todos, etc.). \\
4-Informe final bien presentado y \\
elaborado y buen análisis de la sesión
\end{tabular}} & \multicolumn{4}{|c|}{$\begin{array}{l}\text { 1-Marco teórico bien elaborado. } \\
\text { 2-Algunos problemas en las competencias docentes } \\
\text { durante el desarrollo de la sesión práctica; } \\
\text { 3-Algunos problemas en la presentación oral del } \\
\text { marco teórico (poco clara, leer, no mirar a todos, } \\
\text { etc.). } \\
\text { 4-Informe final bien presentado, pero un análisis } \\
\text { demasiado simple o erróneo de lo sucedido en la } \\
\text { sesión }\end{array}$} & $\begin{array}{l}\text { 1-Marco teórico mal elaborado, incompleto o mal } \\
\text { presentado. } \\
\text { 2-Problemas serios en las competencias docentes durante } \\
\text { el desarrollo de la sesión práctica. } \\
\text { 3-Mala presentación oral del marco teórico (poco clara, } \\
\text { leer, sin preparar, etc.). } \\
\text { 4-Informe final mal presentado, faltan apartados, análisis } \\
\text { demasiado simple o erróneo de lo sucedido en la sesión. }\end{array}$ \\
\hline
\end{tabular}


Pastor, 2009; Manrique et al., 2010; Pérez-Pueyo et al., 2017; Silva \& López-Pastor, 2015).

\section{Conclusiones}

A lo largo de este artículo hemos analizado qué dice la investigación acumulada sobre la importancia de experimentar procesos de EFyC durante la FIP-EF. La razón parece ser doble: (a) las ventajas que tiene la aplicación de estos sistemas de evaluación en sí mismos (aprendizaje, implicación del alumnado, mejora del proceso de enseñanza-aprendizaje, rendimiento académico, etc.); (b) el aprendizaje básico que supone a la hora de desarrollar competencias específicas sobre evaluación y el incremento de posibilidades de que sean capaces de aplicar este tipo de sistemas de evaluación cuando ejerzan como maestros.

Por otra parte, la combinación de metodologías activas y sistemas de EFyC mediante la aplicación de la buena práctica denominada «proyectos de aprendizaje tutorado» resulta muy apropiada para conseguir las ventajas anteriormente citadas, aunque también pueda implicar algunas dificultades y problemas, que pueden ser resueltos. Nuestra intención es continuar utilizando esta metodología, por los buenos resultados obtenidos.

\section{Financiación}

Proyecto «Evaluación de competencias en los Trabajos fin de estudios (Grado y Máster) en la Formación Inicial del Profesorado de Educación Física». Convocatoria: agosto de 2018 del Programa Estatal de I+D+i Orientada a los Retos de la Sociedad en el marco del Plan Estatal de Investigación Científica y Técnica y de Innovación 2017-2020. Referencia: RTI2018-093292-B-I00. Duración: 3 años(2019-2021). Investigadores Principales: María Rosario Romero Martín y Eloísa Lorente Catalán. Universidades de Zaragoza y Lleida.

\section{Referencias}

Atienza, R., Valencia-Peris, A., Martos-García, D., López-Pastor, V., \& Devís-Devís, J. (2016). La percepción del alumnado universitario de educación física sobre la evaluación formativa: ventajas, dificultades y satisfacción. Movimiento, 22(4), 1033-1048. Recuperado de http://www.redalyc.org/html/1153/115349439002

Barba-Martín, J.; López-Pastor, V. M.; Manrique-Arribas, J. C.; GeaFernández, J. M., \& Monjas-Aguado, R. (2010) «Garantir l’èxit en la formació inicial del professorat d'educació física: els projectes d'aprenentatge tutelats». Temps d'educació, 39, 187-206.

Barba, R., \& López-Pastor, V. M. (2017). Buenas prácticas de evaluación en formación inicial del profesorado: los proyectos de aprendizaje tutorados en expresión corporal. Infancia, Educación y Aprendizaje (IEYA), 3(2), 66-70. http://revistas.uv.cl/index. php/ IEYA/index

Barrientos, E.; López-Pastor, V. M., \& Pérez-Brunicardi, D. (2019). ¿Por qué hago evaluación formativa y compartida y/o evaluación para el aprendizaje en EF? La influencia de la formación inicial y permanente del Profesorado. Retos, 36, 37-43. https:// recyt.fecyt.es/index.php/ retos/article/view/66478

Benito; A. \& Cruz, A. (2003). Nuevas claves para la docencia universitaria en el Espacio Europeo de Educación Superior. Madrid: Narcea.

Biggs, J. B. (2005). Calidad del aprendizaje universitario. Madrid: Narcea.

Brown, S. (2015). Learning, teaching and assessment in higher education: global perspectives. London: Palgrave-MacMillan. Cañadas, L., Santos-Pastor, M. L., \& Castejón F. J. (2018). Desarrollo de competencias docentes en la Formación Inicial del Profesorado de Educación Física. Relación con los Instrumentos de Evaluación. Estudios Pedagógicos, 44(2), 111-126. https:// doi.org/10.4067/S0718-07052018000200111

Castejón, F.J., Santos, M. L., \& Palacios, A. (2015). Cuestionario sobre metodología y evaluación en formación inicial en educación física. Revista Internacional de Medicina y Ciencias de la Actividad Física y el Deporte, 15(58), 245-267. https://doi.org/ 10.15366/rimcafd2015.58.004

Córdoba, T., López-Pastor, V. M., \& Sebastiani, E. (2018). ¿Por qué Hago Evaluación Formativa en Educación Física? Relato Autobiográfico de un Docente. Revista Estudios Pedagógicos 44(2), 21-38.

Dochy, F., Segers, M., \& Dierick, S. (2002). Nuevas Vías de Aprendizaje y Enseñanza y sus Consecuencias: una Nueva Era de Evaluación. Revista de la Red Estatal de Docencia Universitaria, 2(2), 13-29. http://revistas.um.es/redu/article/view/ 20051/19411

Estevan, I., Molina-García, J., García-Massó, X., \& Martos, D. (2018). Efecto de la Intervención Docente en la Percepción de Competencia y Motivación de Futuros Maestros de Primaria en Educación Física Utilizando la Evaluación Formativa y Compartida. Estudios Pedagógicos, 44(2), 205-221.

Fernández-Pérez, M. (1989). Así enseña nuestra universidad. Salamanca: Hispagraphis.

Flecha, R., \& Puigvert, L. (1998). Aportaciones de Paulo Freire a la educación y las ciencias sociales. Revista Interuniversitara de Formación del Profesorado, 33, 21-28.

Freire, P. (1990). La naturaleza política de la Educación: Cultura, Poder y Liberación. Barcelona: Paidos-MEC.

Fraile, A., Aparicio, J. L., Asún, S., \& Romero-Martín, M. R. (2018). La evaluación formativa de las competencias genéricas en la formación inicial del profesorado de Educación Física. Estudios Pedadógicos, 44(2), 39-53. http://revistas.uach.cl/index.php/ estped/issue/view/281

Fraile-Aranda, A.; López-Pastor, V. M. \& Castejón-Oliva, F. J. Romero-Martín, R. (2013). La evaluación formativa en docencia universitaria y el rendimiento académico del alumnado. Revista Aula Abierta, 41(2), 23-34.

Fullan, M. (1991). The New Meaning of Educational Change. Cassell: London.

Gallardo, F. \& Carter, B. (2016). La evaluacioìn formativa y compartida durante el praìcticum en la formacioìn inicial del profesorado: anailisis de un caso en Chile. Retos. Nuevas tendencias en Educacioin Fiìsica Deportes y Recreacioìn, 29, 258-263. Recuperado de http://www.redalyc.org/ pdf/3457/345743464048.pdf

Gallardo, F.; Carter, B., \& López-Pastor, V. M. (2019). Evaluación Formativa y Compartida en la Universidad Chilena: Resultados tras cuatro años de implementación. Revista Iberoamericana de Evaluación Educativa, 12(1), 139-155. https://doi.org/10.15366/ riee2019.12.1.008

Gallardo, F.; López-Pastor, V. M., \& Carter (2018). Efectos de la aplicación de un sistema de evaluación formativa en la autopercepción de competencias adquiridas en formación inicial del profesorado. Revista Estudios Pedagógicos, 44(2), 55-77. https://doi.org/10.4067/S0718-07052018000200055

Gibbs, G. (2003). Uso estratégico de la evaluación en el aprendizaje. En Brown, S. y Glasner, A. (Ed.), Evaluar en la universidad. Problemas y nuevos enfoques. Madrid: Narcea, 61-75.

Gutiérrez-García, C. (2018). Informe 2017/2018 sobre la asignatura «Diseño y análisis de la enseñanza de la actividad física», del grado en Ciencias de la Actividad Física y del Deporte. A. RamírezGarcía y M. P. Gutiérrez-Arenas (coord.), XI Congreso Nacional de Evaluación Formativa y Compartida: La evaluación educativa: entre la emoción y la razón. Córdoba, 7-8, septiembre de 2018. Universidad de Córdoba.

Hamodi-Galán, C., López-Pastor, A. T., \& López-Pastor, V. M. (2014). Red de evaluación formativa y compartida en docencia universitaria: creación, consolidación y líneas de trabajo. Revista de eva- 
luación educativa, 3(1). http://revalue.mx/revista/index.php/ revalue/issue/current

Hamodi, C.; López-Pastor, V. M., \& López-Pastor, A.T. (2017). If I experience formative assessment whilst at University will I put it into practice later as a teacher? Formative and shared assessment in Initial Teacher Education. European Journal of Teacher Education, 40(2), 171-190. DOI: 10.1080 02619768.2017.1281909

Hamodi, C., Moreno-Murcia, J. A. \& Barba, R. (2018). Medios de Evaluación y Desarrollo de Competencias en Educación Superior en Estudiantes de Educación Física. Estudios Pedagógicos, 44(2), 241-257.

Hernández-Álvarez, J. L. (2000). La formación del profesorado de Educación Física: nuevos interrogantes, nuevos retos. Tándem, 1, 53-66.

Hortiguela-Alcalá, D., Pérez-Pueyo, Á., \& Abella, V. (2015). ¿Coìmo influye el sistema de evaluación en la percepción del alumnado? @ tic. revista d'innovació educativa, 14, 82-89.

Knight, P. (2005). El profesorado de Educación Superior. Formación para la excelencia. Madrid: Narcea.

López-Pastor, V. M. (2008) Implementing a Formative and Shared Assessment System in Higher Education Teaching. European Journal of Teacher Education, 31(3), 293-311.

López-Pastor, V. M. (coord.) (2009). La Evaluación Formativa y Compartida en Educación Superior: propuestas, técnicas, instrumentos y experiencias. Narcea: Madrid.

López-Pastor, V. M. (2011). Best practices in Academic Assessment in Higher Education: a Case in Formative and Shared Assessment. Journal of Tecnology and Science Education, 1(2), 25-39.

López-Pastor, V. M. (2012) Evaluación Formativa y Compartida en la Universidad: clarificación de conceptos y propuestas de intervención desde la Red Interuniversitaria de Evaluación Formativa. Psychology, Society \& Educatión, 4(1), 113-126.

López-Pastor, V. M. (2013). Nuevas perspectivas sobre evaluación en Educación Física. Revista de Educación Física, 29(3), 1-10.

López-Pastor, V. M., Hamodi, C., \& López-Pastor, A. T. (2016). Evaluación Formativa y Compartida en Educación Superior: buenas prácticas y experiencias desarrolladas por la Red de Evaluación Formativa (pp. 13-44). En Alex Pavié Nova y Mariela Casas Uribe (Editores), Claudio Esparza Freire (Coordinador). Buenas prácticas en evaluación de aprendizajes en educación superior. Ed. Universidad de Los Lagos: Osorno (Chile).

López-Pastor, V. M.; Manrique, J.C.; Vallés, C. (2011). La evaluación y la calificación en los nuevos estudios de Grado. Especial incidencia en la Formación Inicial del Profesorado. Revista Electrónica Interuniversitaria de Formación del Profesorado, 39(144), 57-72. http://www.aufop.com/aufop/uploaded_files/articulos/ 1327436320.pdf

López-Pastor V. M. \& Pérez-Pueyo, A. (coords.) (2017). Evaluación formativa y compartida en Educación: experiencias de éxito en todas las etapas educativas. León: Universidad de León. http://buleria.unileon.es/handle/10612/5999

López-Pastor, V. M.; Pérez-Pueyo, A.; Barba Martín, J. J., \& LorenteCatalán, E. (2016). Rúbricas y PATS. Utilización de una escala graduada (rúbrica) para la autoevaluación de trabajos escritos en la formación inicial del profesorado de educación física (FIPEF). Percepción de su funcionalidad por parte de los estudiantes. Cultura, Ciencia, Deporte (CCD), 31(11), 37-49. DOI: 10.12800/ ccd

Lorente-Catalán, E. \& Kirk, D. (2013). Alternative democratic assessment in PETE: an action-research study exploring risks, challenges and solutions. Sport, Education and Society, 18, 7796. https://doi.org/10.1080/13573322.2012.713859

Lorente-Catalán, E. \& Kirk, D. (2016). Student teachers' understanding and application of assessment for learning during a physical education teacher education course. European Physical Education Review, 22(1), 65-81. https://doi.org/10.1177/ 1356336 X15590352

Lorente-Catalán, E., López-Pastor, V. M., \& Kirk, D. (2018). La evaluación participativa en la formación inicial del profesorado. Un caso sobre su utilización en las primeras experiencias profe- sionales. En E. Lorente-Catalán y D. Martos-García (Eds.), Educación Física y Pedagogía Crítica. Propuestas para la transformación personal y social. Lleida: Edicions de la Universitat de Lleida, 193-213

Manrique Arribas, J.C., López Pastor, V.M., Monjas Aguado, R., \& Real Rubio, F. (2010). El potencial de los proyectos de aprendizaje tutorado y los sistemas de evaluación formativa en la mejora de la autonomía del alumnado. Una experiencia interdisciplinar en formación inicial del profesorado. Revista Española de Educación Física y Deportes, 14, 39-57.

Manrique-Arribas, J., Vallés, C., \& Gea, J. (2012). Resultados generales de la puesta en prática de 29 casos sobre el desarrollo de sistemas de evaluación formativa en docencia universitaria. PSYE Psychology, Society, \& Education, 4(1), 87-102.

Molina, M., López-Pastor, V. M. (2017). La transferencia de la Evaluación Formativa y Compartida desde la Formación Inicial del Profesorado de Educación Física a la práctica real en Educación Primaria. Infancia, Educación y Aprendizaje (IEYA), 3(2), 626-631. http://revistas.uv.cl/index.php/IEYA/index

Molina, M. \& López-Pastor, V. M. (2019). ¿Evalúo cómo me evaluaron en la facultad? Transferencia de la evaluación vivida durante la formación inicial del profesorado a la evaluación practicada como docente. Revista Iberoamericana de Evaluación Educativa, 12(1), 85-101. https://doi.org/10.15366/ riee2019.12.1.005

Palacios, A. \& López Pastor, V. M. (2013) Haz lo que yo digo pero no lo que yo hago: sistemas de evaluación del alumnado en la formación inicial del profesorado. Revista de Educación, 361, 279305. http://www.revistaeducacion.educacion.es/doi/361_143.pdf Pérez-Pueyo, A., Barba-Martín, J.J., López-Pastor V. M., LorenteCatalán, E. (2017). La utilización de escalas graduadas de autoevaluación en la enseñanza universitaria. En: V. M. LópezPastor y A. Pérez-Pueyo (coords.) Evaluación formativa y compartida en Educación: experiencias de éxito en todas las etapas educativas. León: Universidad de León, 374-384. http:// buleria.unileon.es/handle/10612/5999

Romero-Martín, M. R., Asún, S., \& Chivite, M. T. (2016). La autoevaluación en expresión corporal en formacioìn inicial del profesorado de educacioìn física: un ejemplo de buena práctica. Retos. Nuevas tendencias en Educación Física, Deportes y Recreación, 29, 236-241.

Romero-Martín, R., Castejón-Oliva, F. J., \& López-Pastor, V. M. (2015). Divergencias del alumnado y del profesorado universitario sobre las dificultades para aplicar la evaluación formativa. RELIEVE Revista electrónica de investigación y evaluación educativa, 21(1), art. ME5.DOI:10.7203/relieve.21.1.5169.

Romero-Martín, R., Castejón-Oliva, F. J.; López-Pastor, V. M., \& Fraile-Aranda, A. (2017). Evaluación formativa y compartida, competencias docentes comunicativas y TIC en formación inicial del profesorado. Comunicar, 52, 73-82. http://dx.doi.org/ 10.3916/C52-2017-07

Romero-Martín, R.; Fraile-Aranda, A.; López-Pastor, V. M., \& Castejón-Oliva, F. J. (2014). Relación entre sistemas de evaluación formativa, rendimiento académico y carga de trabajo del profesor y del alumno en la docencia universitaria. Revista Infancia y Aprendizaje, 37(1), 16-32. DOI: 10.1080/ 02103702.2014.918818

Santos, M., Martínez, L. F., \& López, V. M. (coords.) (2009). La Innovación docente en el Espacio Europeo de Educación Superior. Editorial Universidad de Almería: Almeria.

Silva, I., \& López-Pastor, V. M. (2015). ¿Cómo vive el alumnado la evaluación en formación inicial del profesorado? @-tic revista de innovación educativa, 14. DOI: 10.7203/ attic.14.4171.

Tejedor, F. J. (Dir.). (1998). Las estrategias utilizadas por los profesores universitarios para la evaluación del aprendizaje de los alumnos. Madrid: CIDE.

Tonucci, F. (2010). Apuntes sobre el manifiesto. Educar(nos), 49, 911.

Zabalza, M.A. (2002). Enseñar en la universidad. Competencias docentes del profesorado universitario. Narcea. Madrid. 